\title{
Successful pregnancy outcome in a case of Takayasu's arteritis with recurrent pregnancy loss with low dose heparin
}

\author{
Richi Khandelwal*, Sudha Gandhi
}

Department of Obstetrics and Gynaecology, RNT Medical College, Udaipur, Rajasthan, India

Received: 20 June 2016

Revised: 17 July 2016

Accepted: 05 August 2016

\section{*Correspondence:}

Dr. Richi Khandelwal,

E-mail: dr_madhu_kh@yahoo.com

Copyright: $\odot$ the author(s), publisher and licensee Medip Academy. This is an open-access article distributed under the terms of the Creative Commons Attribution Non-Commercial License, which permits unrestricted non-commercial use, distribution, and reproduction in any medium, provided the original work is properly cited.

\begin{abstract}
Takayasu's arteritis (TA) is a well-known yet rare form of large vessel vasculitis. TA commonly effect women in childbearing age of Asian origin. The etiology remains speculative but current literature supports autoimmune basis for TA. Suppression of inflammation and preservation of vascular competence form the basis of the treatment. The basic disease appears to be unaffected by pregnancy, inflammatory activity and haemodynamic status improves with pregnancy, but the high arterial pressure and the pre-eclampsia constitute main maternal complications. The fetal complication is intrauterine growth. Therefore, obstetricians are faced with the dilemma of optimal management in pregnancy. The optimum management of these women involves conception during the remission period, antenatal care provided by multidisciplinary team, early detection, treatment of hypertension and curtailing the second stage of labour for better maternal and fetal outcome. In our case, the patient was managed with low dose heparin which was started as soon as the patient visited us (14 weeks) and continued till one day before surgery. No steroids were given at any time during the course of pregnancy and post - one year follow - up the patient is doing fine.
\end{abstract}

Keywords: TA, Low dose heparin, Recurrent pregnancy loss, Steroids

\section{INTRODUCTION}

We present a case report of a pregnant patient who presented to us with early pregnancy after being referred for history of recurrent pregnancy loss. The patient was started on low dose heparin after being diagnosed as a case of takayasu's arteritis and was continued till term. ${ }^{1}$ No steroids were started at any point during treatment. The patient was delivered by elective caesarean section at term, baby was healthy. ${ }^{3}$ Post- 1 year follow - up, the patient is doing fine. ${ }^{6}$ There is paucity of data regarding treatment with only low dose heparin without the use of steroids in the literature, however, in this case the patient did not receive steroids at any time during her treatment. ${ }^{5}$

\section{CASE REPORT}

A 31 year old G7P1A5 with low socioeconomic status came to OPD after being referred from a private hospital due to recurrent pregnancy loss. After getting detailed history patient was subjected to examination however on initial examination her radial pulse rate and BP could not be recorded in $\mathrm{B} / \mathrm{L}$ upper limbs with otherwise no abnormality in rest of the general physical examination. On P/A examination her fundal height corresponded to 14 weeks external ballotment present. She gave history of os tightening one week back. Patient had no complaint but only weakness and past history of abnormal behavior and depression due to repeated losses, diagnosed with schizophrenia for which she was on treatment. Owing to non- recordability of peripheral pulses she was referred to cardiologist where she was diagnosed to be a case of Takayasu's arteritis after being thoroughly evaluated. Doppler report suggested involvement of aorta and its branches (type 3 lesions).

She had a history of one preterm IUD delivery (7 months) $\mathrm{F} / \mathrm{b}$ full term IUD f/b another preterm IUD (8 months) f/b 
preterm delivery (7 months) baby died after 2 days f/b yet another preterm delivery (6 months) baby died. She also had a history of OS tightening in her $4^{\text {th }}$ delivery.

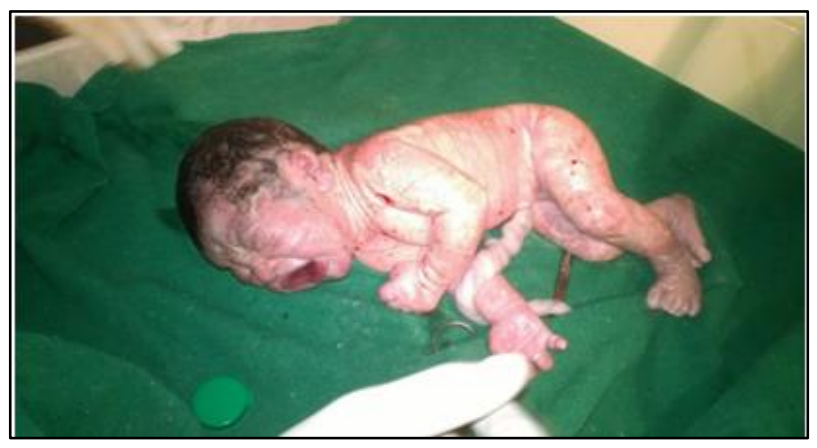

Figure 1: Baby weighing 2500 gm.

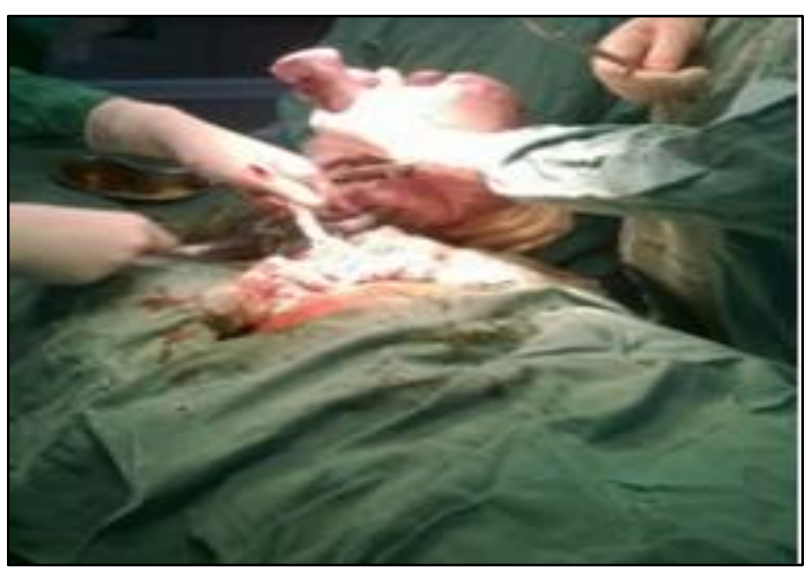

Figure 2: Caesarean section of the patient.

Considering her to be a high risk case she was admitted in the high risk pregnancy ward for safe confinement till delivery.

Her LMP was $21^{\text {st }}$ September 2014. She was started on low dose heparin enoxaparin $40 \mathrm{U}$ s/c daily and tab. Asprin $75 \mathrm{mg}$ OD daily from our side at about 14 weeks GA and was already taking tablet. Olanzapine and tab. Lorazepam as per psychiatrist's advice for auditory hallucination and ideas of persecution which was continued throughout pregnancy as well. Daily FHS monitoring was done and fortnightly ultrasound was done with Doppler to assess fetal well-being every 6 weeks. The patient did not receive steroids in her past treatment $s$ as well as during current management. On examination $\mathrm{b} / \mathrm{l}$ radial and brachial pulses were absent and rest of the peripheral pulses were present and synchronous with normal rate, rhythm and volume.

Her BP was not recordable in B/L upper limbs and was recorded to be normal $(110 / 70 \mathrm{mmHg})$ in $\mathrm{B} / \mathrm{L}$ lower limbs. There was no abnormality detected in respiratory, cardiovascular and nervous system except for psychiatric disturbance. Renal bruit was present bilaterally more in left than right.
Doppler to assess renal and carotid arteries was done in every 8 weeks with minor alterations. Her last Doppler report was normal and cardiologist's opinion was taken on a regular basis and treatment was continued.

The patient tested negative for LA Antibodies but was ANA positive, her ESR was raised moderately; however she was non- reactive for CRP. Her LFT and RFT was mildly derranged at term, protein started appearing in the urine amounting to trace levels as tested by dipstick method after 34 weeks gestation, however there was no rise in $\mathrm{BP}$.

Her fundus examination revealed arterial attenuation, AV crossing, marginal papilledema and was diagnosed to be a case of type 4 retinopathy with macular edema $(r t>1 t)$.

The patient was not given any anti - hypertensive during her pregnancy as her BP was normal limits during daily BP monitoring.

Patient was decided for planned caesarean section at 37 weeks gestational age due to precious nature of the pregnancy and cord around neck which was reported in the last Doppler for fetal well being

Low dose heparin was stopped one day before surgery as per anesthetist's advice. Patient delivered a healthy baby with 2500 gm weight with Apgar score of 8 at 1 and 9 at 5 minutes. She was discharged on $8^{\text {th }}$ postoperative day.

Post 1 month follow - up the patient has been doing well and has improved with her psychiatric symptoms and taking good care of herself and her baby.

\section{DISCUSSION}

Takayasu's disease (also known as "aortic arch syndrome," "nonspecific aortoarteritis," and "pulseless disease") is a form of large vessel granulomatous vasculitis with massive intimal fibrosis and vascular narrowing, affecting often young or middle-age women of Asian descent. ${ }^{1,2}$ It mainly affects the aorta (the main blood vessel leaving the heart) and its branches, as well as the pulmonary arteries. Females are about 8-9 times more likely to be affected than males. ${ }^{2}$

Takayasu Arteritis was first described in 1908 by two Japanese ophthalmologists, Takayasu and Onishi, who observed retinopathy in the absence of peripheral pulses. The cause is unknown, but it seems to be related to autoimmunity, sex hormones (more common in young females), and genetics (demonstrated by the predisposition of the human leukocyte antigen - HLA BW 52). ${ }^{2}$ Four types of Takayasu arteritis can be identified: type I (disease involving the aortic arch and its branches), type II (lesions restricted to descending thoracic aorta and abdominal aorta), type III (patients have characteristics of types I and II), and type IV (involvement of the pulmonary artery). ${ }^{1}$ The disease can 
also be classified into stages according to the presence of major complications such as hypertension, retinopathy, aneurysms, and aortic insufficiency. ${ }^{1}$ Stage I, no complications are observed; stage II a, patients have only one of these complications; and stage II b, patients have only one of these complications, but the severe form; stage III, when more than one complication is present. The patient presented here was in stage II, but during pregnancy her hypertension was compensated. ${ }^{4}$ Diagnosis is based on signs and symptoms, inflammatory markers, and arteriography demonstrating aortic stenosis and of its branches. ${ }^{1}$ However, the gold-standard for diagnosis is the biopsy of the vessel.

Pregnancy does not interfere on disease progression, although hypertensive complications such as preeclampsia and exacerbation of chronic hypertension, and fetal complications such a restriction of intrauterine growth, abortion, and fetal death have been reported in $60 \%$ to $90 \%$ of the cases. ${ }^{4,6}$ The high incidence of restricted uterine growth seems to be related to uncontrolled hypertension and aorta and iliac arteries involvement. Arterial ultrasound doppler, which quantifies the flow in the uterine arteries, is another way of evaluating fetal well-being and growth in patients with TA. Few patients are asymptomatic and more than $60 \%$ have some kind of complication. Participation of other specialties is fundamental for detection and treatment of disease complications. In the case presented here the disease was compensated with drug therapy, and strict rest.

Conventional treatment of TA involves the use of corticosteroids, the first line treatment for inflammatory activity as well as immunosuppressors including methotrexate and azathioprine. ${ }^{5}$ Although in this case the patient did not receive any steroids during the course of her treatment. These questions the basic etiology of this disease whether it is really an autoimmune disease. ${ }^{2}$ However, intraoperative corticosteroid supplementation was done with intravenous hydrocortisone. ${ }^{5}$

A study from Hong Kong in reported on 13 women who had experienced a total of 30 pregnancies. $^{3}$ Apart from hypertension, there were no major obstetric problems and no maternal deaths directly related to pregnancy. Fetal outcome could be predicted on the basis of maternal vessel involvement (abdominal aorta and renal), severity of maternal hypertension, superimposed pre-eclampsia, and timing of adequate blood pressure control.

Maternal complications reported in 12 patients from India included superimposed pre-eclampsia, congestive cardiac failure, progressive renal impairment, and one case of postpartum sepsis. Abdominal aortic involvement and a delay in seeking medical attention predicted a poor perinatal outcome.

Fertility is not adversely affected, pregnancy per se does not appear to exacerbate the disease, but management of hypertension is essential. Hypertension in the second stage of labor is a risk factor for cerebral haemorrhage; shortening this stage by use of low forceps delivery or vacuum extraction appears to be a reasonable solution. ${ }^{3}$

Vaginal delivery could be indicated for patients in groups I and II a, as long as epidural analgesia is used for pain relief, and delivery is abbreviated by the use of forceps especially in hypertensive patients. In groups II b and III, one should prefer caesarean section, since the increased blood volume and blood pressure observed during uterine contractions as well as the increased cardiac output observed during labor may lead to cardiac decompensation. $^{3}$ Although our patient was haemodynamically stable and belonged to group II b, she underwent caesarean section because of the precious nature of her pregnancy.

Post-one year follow - up the patient is doing fine without any steroids or anti - hypertensives., ${ }^{4,5}$

Funding: No funding sources

Conflict of interest: None declared

Ethical approval: The study was approved by the Institutional Ethics Committee

\section{REFERENCES}

1. Ishikawa K. Natural history and classification of occlusive thromboaortopathy (Takayasu's disease). Circulation. 1978;57:27-35.

2. American college of physicians (ACP). Medical Knowledge Self-Assessment Program (MKSAP-15). Rheumatology. Systemic Vasculitis. 2009:65-67.

3. Wong VCW, Wang RYC, Tse TF. Pregnancy and Takayasu's arteritis. Am J Med. 1983;75:597-601.

4. Sharma BK, Jain S, Vasishta K. Outcome of pregnancy in Takayasu arteritis. Int $\mathbf{J}$ Cardiol. 2000;75:S159-62.

5. Mitchell K, Kaul M, Clowse ME. The management of rheumatic diseases in pregnancy. Scand $\mathbf{J}$ Rheumatol. 2010;39:99-108.

6. Gayed M, Gordon C. Pregnancy and rheumatic diseases. Rheumatology (Oxford). 2007;46:34-1640.

Cite this article as: Khandelwal R, Gandhi S. Successful pregnancy outcome in a case of Takayasu's arteritis with recurrent pregnancy loss with low dose heparin. Int J Reprod Contracept Obstet Gynecol 2016;5:3210-2. 